\title{
Developing Discriminative Requesting Using Natural Environment Training in Two Children with Autism Spectrum Disorder
}

\author{
Nouf M Alzrayer ${ }^{*}$ and Devender $R$ Banda $^{2}$ \\ ${ }^{1}$ Department of Special Education, College of Education, King Saud University, Riyadh, Saudi Arabia \\ ${ }^{2}$ Department of Educational Psychology and Leadership, College of Education, Texas Tech University, \\ Lubbock, Texas, USA
}

*Corresponding author: Nouf M Alzrayer, Ph.D., BCBA, Assistant Professor, Department of Special Education, College of Education, King Saud University, PO Box 266, Riyadh 11567, Saudi Arabia, Tel: +96614670000, Fax: +96614677580, E-mail: nalzrayer@ksu.edu.sa

\begin{abstract}
The use of touchscreen devices for communication purposes has been shown to be effective in the literature; however, there is little research in the effectiveness of applying naturalistic teaching approaches in developing discriminative requesting in young children with autism spectrum disorder (ASD). Two participants between the ages of 3 and 5 , who were non-vocal, were taught to select a target symbol when presented with varying number of non-target symbols. Behavioral therapists who frequently interacted with the participants were trained to implement the intervention during playtime within their natural environment. We used a multiple probe design across different field sizes to evaluate the effects of the intervention. Results revealed that the symbol discrimination training and naturalistic teaching methods yielded high-to-medium effects on the acquisition of discriminative requesting and the generalization of such skills across novel stimuli.
\end{abstract}

\section{Keywords}

Autism spectrum disorder, Augmentative alternative communication, Speech-generating devices, Discriminative requesting, Tablets

Speech and language developmental delays are prevalent among children with autism spectrum disorder (ASD) [1]. These deficits may impact the children's ability to communicate both verbally and nonverbally [1]. A significant body of literature exists pertaining to the use of aided augmentative alternative communication (AAC) systems to teach communication skills in children with ASD- to overcome such deficits [2]. Aided AAC systems, as a subcategory under the umbrella of
$A A C$, require the use of external devices, which range from low-tech (e.g., picture exchange communication system [PECS]) to high-tech (e.g., laptops with synthesized speech output, tablets with dedicated AAC applications), to convey a message [3].

Speech-generating devices (SGDs) have become a prominent method for individuals with ASD [4]. Evidence suggests that SGDs have a significant impact on improving functional communication skills in children with ASD $[5,6]$. Touchscreen devices have opened a new avenue in the AAC field that enables the use of such sophisticated computers such as dedicated SGDs. Empirical evidence has shown that iPads or iPods with AAC applications are effective in improving functional communication skills that vary in their complexity from a simple single-step request (i.e., selecting 1 symbol) to more advanced multistep responses (i.e., activating multiple symbols across different pages) [7]. Beside requesting, iPad/iPod Touch has been used successfully in improving labeling $[8,9]$ and answering questions [10].

The ultimate objective of AAC interventions is for individuals to functionally communicate and socially interact with others in their natural environment [11]. The literature has documented the use of either teacher-lead (i.e., discrete trial training; DTT) or a child-directed teaching format (i.e., natural environment training; NET) to attain this goal $[2,12]$. The foremost distinction between these two teaching approaches is that NET uses a child's current interests or motivation to teach 
a variety of communication skills, especially manding/ requesting [13]. Natural environment training, as a teaching procedure, requires that an adult or the teacher conduct the following steps: (a) Enrich the environment with the child's preferred items, (b) Wait for the child to demonstrate interest by reaching or pointing at a preferred item, (c) Prompt the target response when needed, and (d) Reinforce the occurrence of the target response (e.g., pointing to a picture on a commutation book, selecting a symbol on a display screen) by delivering the desired item.

Adapting naturalistic teaching approaches (e.g., incidental teaching, time delay, NET) in developing language skills has several advantages compared with more structured and systematic teaching strategies. The primary advantage is that such teaching strategies help to facilitate the use of language across novel circumstances within the natural environment and reduce the disruptive behaviors that typically occur to avoid or escape aversive situations [13-15]. Natural environment training has been used in the literature to teach different verbal behaviors such as, manding for information and manding for missing items $[16,17]$. The investigators of the previously cited studies manipulated the environment to motivate the participants to request specific reinforces or tact items/actions.

A small number of studies have utilized naturalistic teaching approaches to train individuals with ASD to use tablets for communication purposes [18-20]. In these three studies, the interventions were implemented during snack time [18], playtime $[19,20]$ and by the participants' common communication partners (i.e., parents, teachers, behavioral therapists) [18]. However, the teaching method was child-led in only two of the studies $[19,20]$, meaning that the instruction began when the child demonstrated an interest in an item by reaching, trying to grab, or gazing at it.

Another fundamental objective of communication interventions is to expedite the development of language acquisition of individuals who use AAC that it will be comparable to their peers with typical development. The display design of AAC modalities may enhance the ability of these individuals to reach such a level [21]. However, many studies that focused on using tablets as dedicated SGDs were limited in that the display design of the device would not permit individuals with ASD to effectively communicate [22]. For example, the participants in most studies were taught to select a symbol to express general request (e.g., I want more) when the display design depicted a single symbol and other distractors or blank icons $[20,23]$. The other studies that configured the tablet to display a more extensive field size (i.e., number of symbols on the display screen) did not directly expose their participants to symbol-discrimination training prior to teaching augmented communication skills [18].
Discrimination training plays a vital role in the systematic teaching of skills in different domains such as communication, social, academic, and daily living skills. Such training refers to the reinforcement of a behavior when it occurs under specific circumstances, but not in others [24]. For example, a child selects a symbol of a toy when there is a picture that corresponds to the toy in view and does not select symbols of other referents. Several instructional strategies have been reported to be effective at establishing discrimination skills: Stimulus prompting (e.g., altering specific features of a discriminative stimulus [25]) and response prompting (e.g., assisting the individual to respond correctly to the discriminative stimulus [26]).

To date, there have been only two studies that have directly focused on teaching symbol discrimination when using tablets for requesting $[19,27]$. Lorah and colleagues (2014) [27] used within-stimulus prompting and prompt fading to teach four preschool children with ASD to discriminate between the target symbol (i.e., a picture of a preferred item) and other distractors/ non-target symbols (i.e., neutral items). The procedure focused on symbol discrimination training by gradually increasing the filed size of preferred, neutral, and non-preferred or blank symbols on the display screen. Overall, the study showed viable findings on the efficacy of the training procedure to teach symbol discrimination using the iPad to request a specific item. There were, however, few areas of concern in this study include lack of generalization probes, social validity, and natural teaching procedure.

Similarly, Lorah (2016) [19] expanded the previously conducted study [27] by slightly modifying the symbol discrimination training and conducting the sessions within the natural setting. The investigator used constant-time delay and full-physical prompting to teach three children with ASD, between the ages of 3 and 4 years, to select the target symbols that corresponded to preferred items when being presented with a larger field size (a total of 4 symbols of preferred items). The result indicated that the training procedure was effective in developing discriminative requesting repertoire in young children with ASD. One of the primary limitations of this study was the use of less rigorous mastery or acquisition criteria ( $80 \%$ or higher of independent and accurate requesting across two sessions) to transition from one phase to the other.

The present study aimed to expand upon previous studies $[19,27]$ and to contribute to the literature by focusing on modifying the PECS Phase III to teach general/generic and discriminative requesting (i.e., selecting play symbol to access different preferred toys) in a natural environment. Generic requesting was targeted to keep the symbols and the field size consistent and to prevent the need to change the field size based on the participants' dynamic preferences. In addition, the use 
of generic symbols instead of pictures that correspond to preferred items allows adding potent reinforcers and remove less preferred items, which result in keeping the participants' motivated to request to play throughout the study. Therefore, the purpose of the study was to determine whether (a) Applying naturalistic teaching approaches will facilitate the development of discriminative requesting in young children with ASD, (b) Utilizing modified version of PECS Phase III training procedure will improve symbol discrimination when using an iPad-based-SGD for requesting, and (c) Generalizing of discriminative requesting will occur across other preferred items and behavioral therapists.

\section{Method}

\section{Participants}

A local applied behavior analysis (ABA) therapy service provider in the South-Central region of the United States was screened to recruit participants for the study. Out of 22 children with ASD and/or developmental disabilities, two participants, between the ages of 3 and 5, meet the following inclusion criteria: (a) A diagnosis of ASD made either by a medical professional or a school psychologist, (b) Absent or weak mand repertoire based on the Verbal Behavior-Milestones Assessment and Placement Program [28] Barriers Assessment, (c) No visual and/or hearing impairments that would prevent the participant from manipulating an iPad, and (d) No previous history of using an iPad with Proloquo2Go. Informed consent was obtained from all individual participants included in the study. Refer to Table 1 for information about each participant's communication skills characteristics.

Erin was a three-year-old boy diagnosed with ASD. He did not use vocal communication functionally and frequently engaged in challenging behaviors (e.g., screaming, crying, hitting, biting) as a means of communicating wants or rejecting aversive or undesirable stimuli. Prior to the study, Erin was receiving training on the use of PECS Phase IIIB. However, he was having difficulty reaching mastery in this Phase, especially locating the picture card that corresponded to the preferred item. He needed hand-over-hand prompting to discriminate between pictures (a mixture of colored line drawings and real photographs) of preferred and non-preferred items when using the communication book for requesting. One of Erin's goals in his current program was to independently request eight items using pictures or vocalizations when preferred items were presented to him.

Jacob was a five-year-old boy diagnosed with severe ASD. He was not able to use vocal communication functionally; therefore, he often engaged in self-injurious behaviors (e.g., head banging) and aggression toward others (e.g., hair grabbing) to express wants, especially when his prelinguistic communication forms were not understood by unfamiliar people. Jacob had prior exposure to PECS Phases IIIB at the time of the study. However, he had difficulty reaching mastery in this Phase. He was not able to discriminate between target symbols and non-target (i.e., distractors). Besides, Jacob engaged in self-stimulatory behavior while holding the picture card after locating it on the communication book. He tended to flap the picture card on the tip of his fingers repeatedly instead of reaching to the communication partner and releasing the picture card. One of Jacob's goals on social-communication skills was to use up to two words to request using total communication forms, such as vocalizations, word approximations, signs, gestures, and picture exchange.

\section{Setting}

The study took place in ABA therapy center, specifically in a play area where the participants typically spent their leisure time playing with their behavioral therapists. The placement was chosen for two reasons: (a) To allow the participants to receive natural contingencies [31] and (b) To enhance generalization of discriminative requesting [32]. The session's length was 5 min long, conducted twice per day, four times per week, over the course of 12 weeks ( 48 days in total). There was a $5 \mathrm{~min}$ break between consecutive sessions. The format of the session was child-directed, meaning that the child led the session by freely selecting preferred toys. Baseline and intervention sessions were videotaped to collect reliability and treatment integrity data.

\section{Experimental design}

We used single-case experimental design [33], specifically a multiple-probe design [34] across different field sizes (i.e., 1, 2, 4 symbols) to determine the impact of the

Table 1: Participants communication skills characteristics.

\begin{tabular}{|c|c|c|}
\hline Participants & Erin & Jacob \\
\hline${ }^{1}$ Possibility of ASD & 89 (very likely) & 100 (very likely) \\
\hline Prelinguistic skills & $\begin{array}{l}\text { Gestures, vocalizations, word } \\
\text { approximations (e.g., pa, by) }\end{array}$ & $\begin{array}{l}\text { Gestures, vocalizations, word } \\
\text { approximations (e.g., bye, hi) }\end{array}$ \\
\hline \multicolumn{3}{|l|}{ ²VP-MAPP } \\
\hline Milestone & Level 1 & Level 1 \\
\hline Barrier & 3 (Weak) & 4 (Absent) \\
\hline \multicolumn{3}{|l|}{${ }^{3}$ ABAS-2 } \\
\hline Communication domain & Extremely low & Extremely low \\
\hline
\end{tabular}

Note: ${ }^{1}$ Autism Index (AI) score on Gilliam Autism Rating Scale-Third Edition (GARS-3 [29]); ${ }^{2}$ Verbal Behavior-Milestones Assessment and Placement Program (VB-MAPP [28]); ${ }^{3}$ Adaptive Behavior Assessment System-Second edition (ABAS-2 [30]). 


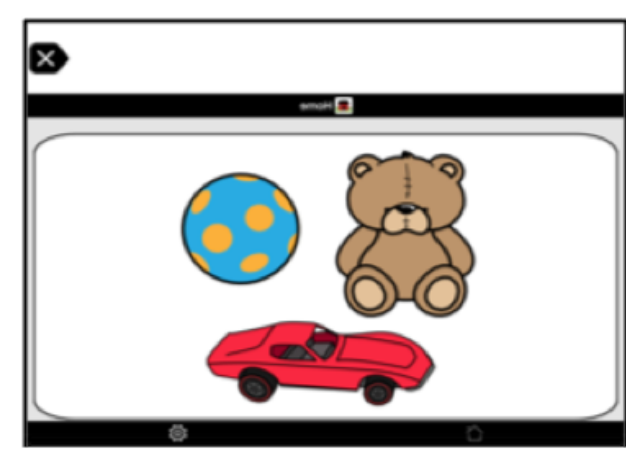

1 symbol

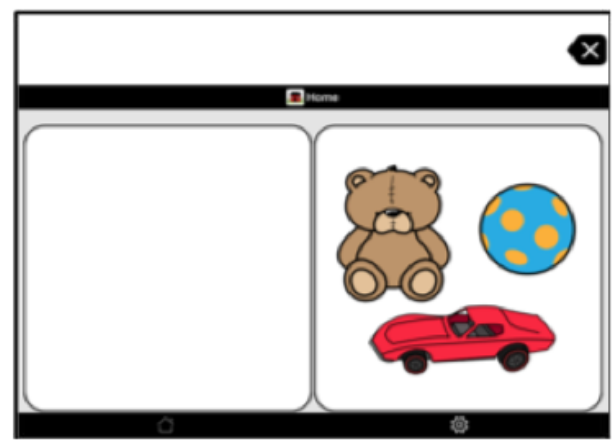

2 symbols

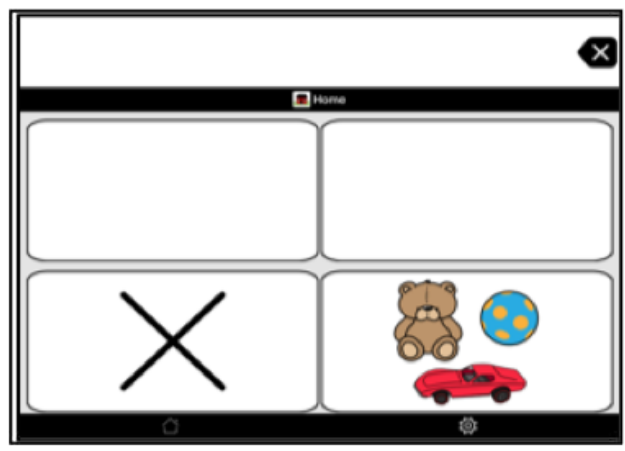

4 symbols

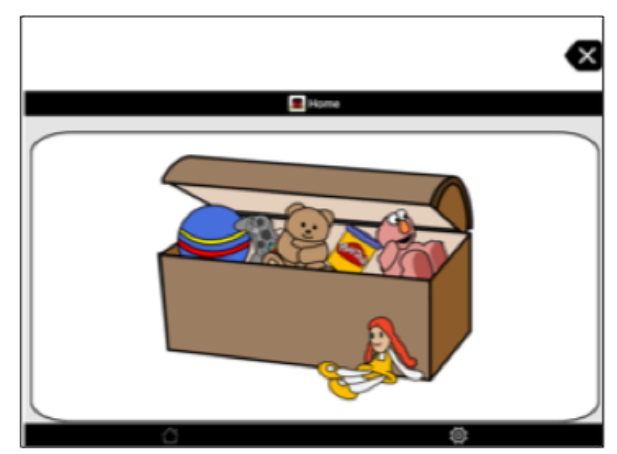

1 symbol

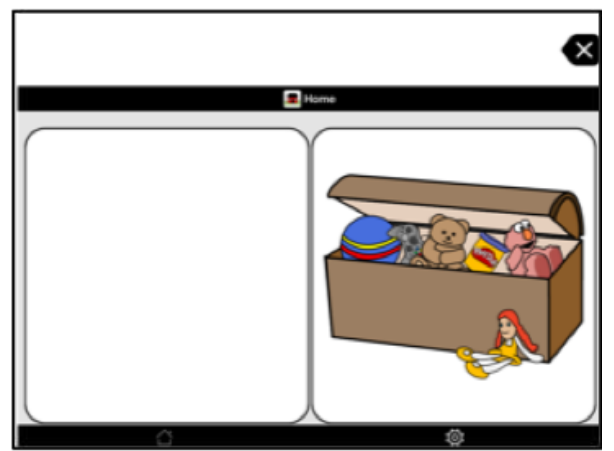

2 symbols

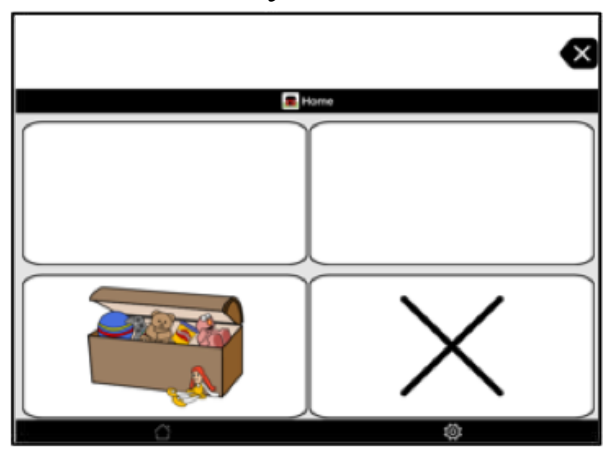

4 symbols

\section{(A) Intervention}

(B) Generalization

Figure 1: Display screen across different field sizes for intervention and generalization phases.

intervention. Baseline probes were collected for the single symbol field size for both participants. When the baseline data reached stability (i.e., no more than $5 \%$ variability on the dependent measure), the intervention was introduced for the single symbol field size till the participants met the acquisition criteria (i.e., $80 \%$ or higher across three consecutive days). The intervention was then introduced across the other field sizes in the same manner as the previous field size. Before the implementation of the intervention on the 2 and 4 symbols field sizes, baseline probes were conducted to assess the participants' ability to discriminate between symbols in a larger field size.

\section{Materials}

A 16GB Apple iPad II loaded with Proloquo2Go application [35] was used as a dedicated SGD. The iPad was configured based on the different number of symbols in each field size. The target symbol (i.e., play) in all field sizes showed a colored line drawing of a brown teddy bear, a ball, and a red car toy. Selecting the symbol activated the synthetic voice output that labeled the icon "play" (See Figure 1). The synthetic voice output for the other non-target symbols was inactive, meaning that touching them did not produce the synthetic voice output. During generalization, the configuration was the same for the baseline, intervention, and maintenance sessions. However, the target symbol was a colored line drawing of a brown box with several toys. It was done to assess the participants' ability to generalize symbol discrimination across different symbols. The location of the symbols as well as the placement of the iPad were rotated prior to the start of each session to (a) Prevent position bias of symbol or iPad selection and (b) Prompt scanning the array before activating the target symbol. The target and the non-target symbols displayed pictures only without accompanying texts.

\section{Modified PECS phase III}

The typical procedure used in PECS Phase III was 
modified to promote the acquisition of discriminative requesting using high-tech SGDs. To reach mastering for PECS Phase III, the learner is required to do the following: (a) Reach for the communication book, (b) Scan the arrays of symbols that correspond to preferred and non-preferred items, (c) Locate the picture card of the preferred item, (d) Pick up the card and hand it to the communication partner. We have aimed to lessen the response effort of the previous procedure by changing the topography of the access method (i.e., touching instead of pulling and handing a picture card) and the type of symbols (i.e., general instead of specific for each object). To access preferred items during play, the participants must reach for the iPad, scan the array, find and activate the target symbol correctly and independently.

\section{Data collection methods}

The number of independent and prompted discriminative requesting was counted as the primary dependent measures. Independent discriminative requesting was defined as activating the play symbol on the page screen by touching the icon once with enough pressure using the tip of the fingers to produce the synthetic voice output. Discriminative requesting was considered prompted when it occurred within 1-5 s of gestures, verbal, and physical prompts provided by the behavioral therapists. Further, incorrect responses were also measured throughout the study to analyze the type of errors that the participants frequently exhibited. Responses were considered incorrect if they fell into one or more of the following categories: (a) Random selection- randomly tapping the main screen; (b) Inactivation- touching the symbol without enough force to activate the synthetic voice output; (c) Multiple selection- tapping a symbol multiple times; and (d) Distractors- selecting other distractor/non-target symbols.

\section{Training behavioral therapists}

The first author trained the behavioral therapistswho delivered instructions to the participants - to conduct the baseline, intervention, and generalization sessions. The training consisted of the following: (a) Explaining the purpose of the study, the target response, and the procedure across all phases; (b) Presenting correct and incorrect examples of the procedures and the target response; and (c) Role-playing the procedures for all phases, which occurred between the first author and the behavioral therapists. Following the training, the behavioral therapists used the treatment integrity checklist to evaluate the accuracy of the procedure implementation during the role play during baseline and intervention. Once the behavioral therapists attained $100 \%$ accuracy on the treatment integrity, baseline sessions were conducted.

\section{Preference assessment}

Using the Indirect Preference Assessment Interview
Protocol [36], parents and behavioral therapists were interviewed, either in person or via the phone, to determine the participants' preferred items. The suggested lists of preferred toys were used to conduct direct preference assessment.

For direct preference assessment, a contrived free-operant preference assessment was conducted to select each participant's preferred toys [37]. The behavioral therapist presented a single toy, one at a time, for each participant to sample for roughly $15-25 \mathrm{~s}$. After sampling, a total of 3-5 toys were placed randomly on the floor, and the participant was told to play with the toys. Three elements were recorded during the sessions: Approach (e.g., reaching, eye gazing), engagement (e.g., manipulating the toys), and duration (i.e., time spent interacting with each toy). The sessions were 5 min long and conducted 2-3 times per day across four consecutive days. The results of the preference assessment indicated that Erin's preferred toys were music toys, sensory activities (e.g., dry rice or pasta in a sensory bin), and music videos. Jacob, on the other hand, mainly preferred sensory and fidget toys (e.g., tangle toy, spinners, ball). These items were used during baseline, intervention, and maintenance phases.

\section{Procedure}

Baseline: Each baseline session consisted of 10 trials where the participants were given an opportunity to initiate a communication using the iPad. Prior to each session, the iPad, which was openly displaying the main page that showed the play symbol, was placed within the participants' reach. The behavioral therapists commenced the sessions by structuring the environment to include several of the participants' items. Each trial began by conducting in-vivo preference assessment. The assessment was conducted by placing 4 to 6 preferred toys randomly around the play area, either on the ground or at the table, during playtime. The behavioral therapists instructed the participants to play with the toys. When the participants emitted a behavior indicator (e.g., reading, pointing, gazing) or an interest to play with a toy, all other toys were removed, and they were allowed to play with the selected toy for at least $5 \mathrm{~s}$. Then, the behavioral therapists created an opportunity for the participants to initiate a request by gently taking away the selected toy for $5 \mathrm{~s}$ and while saying, " $m y$ turn". If the participants initiated a request by pressing the "play" symbol on the iPad during the 5-s interval, the behavioral therapists said nothing and gave back the toy to the child by the end of the interval. For incorrect and no responses that occurred during the 5-s interval, the behavioral therapists said nothing and gave the participants access to the toys by the end of the interval. The participants were given $15 \mathrm{~s}$ to play with the selected toy for correct, incorrect, and no responses. The reason for applying fixed ratio schedule of natural reinforcement was to keep the participants motivated 
to interact with the therapists throughout the session. The behavioral therapists played with the participants during the $15 \mathrm{~s}$ to maintain a natural context. During this phase, the behavioral therapists did not provide prompts (e.g., verbal, gestural, physical) verbal praise or social reinforcements.

Intervention: The procedure was identical to the baseline, except that the behavioral therapists provided most-to-least prompting, progressive-time delay, and differential reinforcement to teach the participants to use the iPad to request to play with preferred toys [38]. Also, prompting was delivered, when needed, after the participants attempted to reach for the toy during the 5 $s$ interval to ensure that participants were motivated to initiate a request using the iPad.

$\mathbf{0}$ s-delay: The first four sessions of the intervention for each field size, the behavioral therapists provided immediate, full physical prompts by holding the participant's hand to select the play symbol when the interruption occurred. When the participants activated the target symbol with hand-over-hand prompting, the behavioral therapists provided verbal feedback by saying, "that is right, you want to play!" and gave them $20 \mathrm{~s}$ to play with the selected toy (i.e., fixed interval schedule of reinforcement). Also, the behavioral therapists provided verbal praise (e.g., good job asking) and social reinforcement (e.g., high fives, tickles) for the prompted responses.

1-5 s delay: The behavioral therapists provided partial physical prompt (i.e., slightly touching the participant's wrist) when no response occurred over 1 to $5 \mathrm{~s}$ interval. In the first two trials, the behavioral therapists delayed the delivery of the prompts by $1 \mathrm{~s}$, then the delay increased by an additional $1 \mathrm{~s}$ in the subsequent set of trials until reaching a terminal delay of $5 \mathrm{~s}$. If the participants initiated the discriminative requesting independently prior to the delivery of the prompts, the behavioral therapists immediately delivered the toy to play for $25 \mathrm{~s}$ and said, "that's right, you want to play!" For prompted responses, the behavioral therapists said nothing while giving the selected toys back to the participants and gave them $20 \mathrm{~s}$ to play. When the participants made errors using the iPad to request, the behavioral therapists used full-physical prompts to ensure the occurrence of the target response. For incorrect discriminative requesting, the behavioral therapists gave the participants $20 \mathrm{~s}$ to play with the selected toy and said nothing.

When the participants used the iPad to perform the discriminative requesting independently for at least $50 \%$ of the trials across four consecutive sessions, the intrusiveness of the prompt decreased further from partial-physical to verbal (i.e., providing verbal instruction to touch the play symbol) and gestural (i.e., pointing to the target symbol) prompts. Further, verbal and social reinforcements were provided only for indepen- dent discriminative requesting when the participants independently used the iPad to request at least $50 \%$ of the trials across four consecutive sessions.

Generalization: Pre-and post-intervention generalization probes were conducted to test the participants' ability to generalize discriminative requesting using the iPad across new preferred toys and other behavioral therapists who frequently interact with the participants. Erin's preferred items that were tested for generalization were the following: Slide ball baby rattle toy, kinetic $^{\mathrm{TM}}$ sand, and water beads. Jacob's preferred toys that were used during this phase were liquid timers, and pin impression toy, and glitter wand. The procedure was identical to the one that was used during the baseline. Probes were implemented twice per week over a threeweek period. There was a one-week gap between the last intervention session and the first generalization probe to prevent the possible carry-over effect.

Maintenance: The probes were carried out using the same baseline procedure to assess the participants' ability to maintain the acquired discriminative requesting using the iPad postintervention phase. There was a two-week gap between the last generalization probe and the first maintenance probe. The probes were conducted twice per week for two weeks.

\section{Interobserver agreement}

To check the reliability of the collected data, an independent observer (a graduate student with a background in $A B A$ ) reviewed the videotaped baseline and intervention videotaped sessions. The reliability data were collected for $33 \%$ of the videotaped sessions for each baseline, intervention phase for each participant. A trial-by-trial method was used to calculate the agreement by summing the number of agreements and dividing that number by the total number of agreements and disagreements multiplied by 100 . For Erin, the agreement was $100 \%$ for the baseline and intervention sessions across different field sizes. Similarly, the agreement for Jacob's baseline sessions was $100 \%$ across all field sizes, and the intervention sessions averaged 100, 99, and 87 for symbol 1, 2, and 4, respectively.

\section{Procedural fidelity}

The first author checked the procedural fidelity using two separate checklists (baseline, intervention) live for $100 \%$ of the sessions in each phase for both participants. The procedural fidelity was calculated by dividing the number of correctly performed steps by the total number of steps multiplied by 100 . The results yielded an average of 99, 96, 96, 100, and 100\% for Erin and 100, $95,95,96$, and $97 \%$ for Jacob across the baseline, a 0-s delay, a 1-5-s delay, generalization, and maintenance, respectively. Furthermore, the independent observer, who collected the interobserver agreement (IOA) data, also checked the IOA on the procedural fidelity. The independent observer reviewed the same videos that 


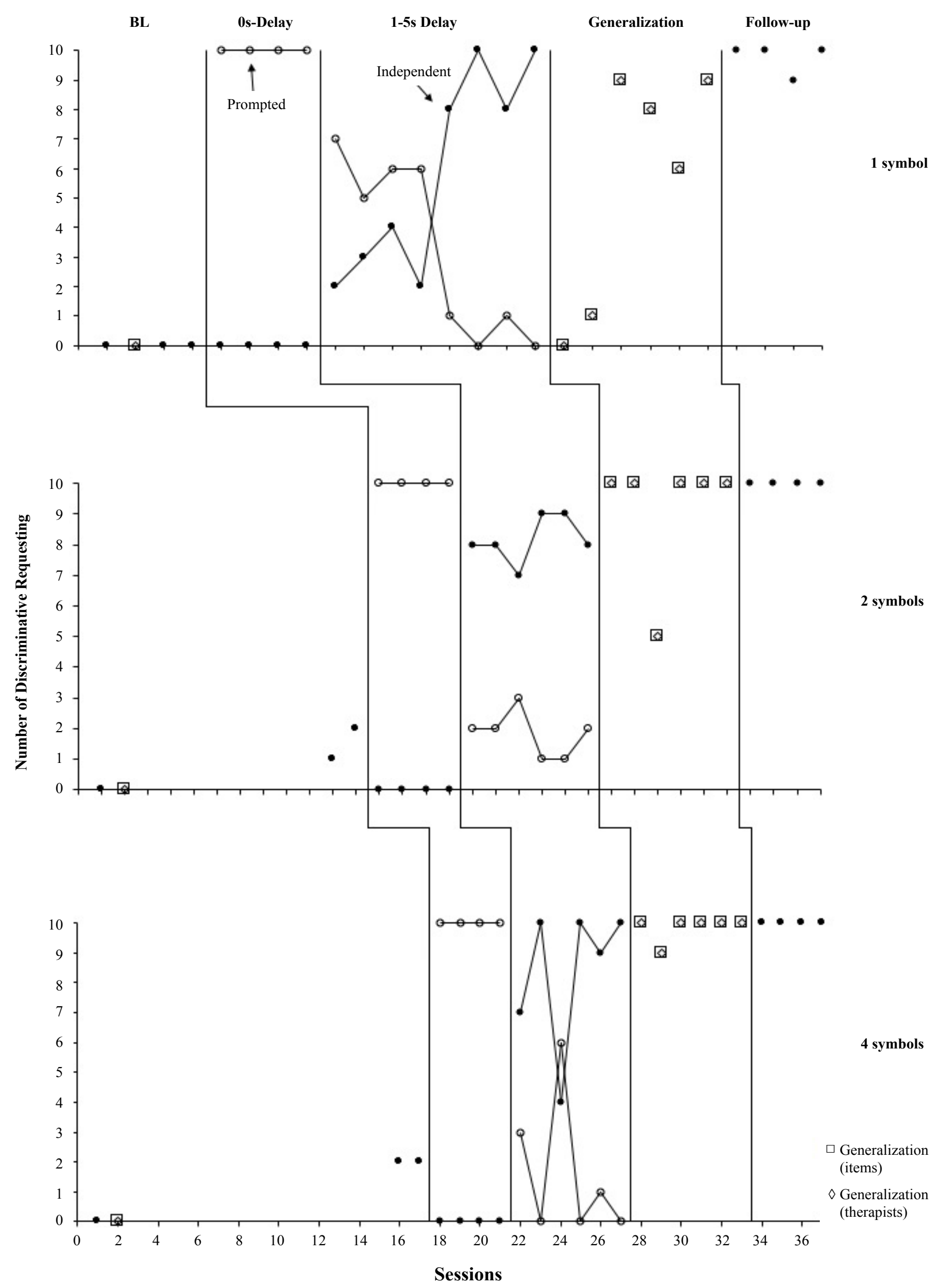

Figure 2: Number of independent requesting across different field sizes for Erin.

were used for calculating the IOA. The agreement between the first author and the independent observer was $100 \%$ for the baseline and intervention sessions across different field sizes and participants. 


\section{Social validity}

The social validity of the study was assessed using a questionnaire that contained several statements rated using a 4-point Likert scale ranging from 1 (strongly disagree) to 4 (strongly agree). The behavioral therapists, who implemented the intervention, reported that manding was deficient in the participants' repertoire and that targeting such skills was critical; the average rating was 4 (strongly agree). The behavioral therapists also agreed that the participants exhibited improvement in independent manding after the implementation of the intervention and that the change was socially significant (average rating: 3.9 [agree]). Similarly, the behavioral therapists reported that the intervention was both practical and cost-efficient (average rating: 3.3 [agree]). Concerning ecological validity, the behavioral therapists reported their willingness to use the intervention after the discontinuation of the study (average rating: 3.5 [agree]).

\section{Results}

We used visual analysis to determine the degree of experimental effects of the intervention. Figure 2 and Figure 3 illustrate the participants' performance in both prompted and independent discriminative requesting. In addition, we used Tau- $U$ to measure the magnitude of the intervention's effectiveness [39]. Tau-U, as a nonparametric effect size, overpowers other nonoverlap indices in that it considers the intervention trend and provides the option to control unwanted baseline trend when measuring the lack of overlap between two phases [39]. The weighted average Tau- $U$ effect size was 1.00 with a $95 \%$ confidence interval $(\mathrm{Cl})(0.50$, $1.00), 0.98$ with $95 \% \mathrm{Cl}(0.51,1.00)$, and 0.85 with $95 \%$ $\mathrm{Cl}(0.31,1.00)$ for the filed size of 1,2 , and 4 symbols, respectively. The results indicated a medium-to-strong effect and statistical significance $(p=0.00)$ for all three phases.

\section{Erin}

1 symbol: Erin did not select the play symbol when his play was interrupted. He met the acquisition criteria after the seventh session and averaged 5.88 (standard deviation $(S D)=3.48$ ). The data revealed a gradual change in level, positive trend, and high variability. There was no overlap between the baseline and the intervention data points. When the intervention was implemented, the data showed an immediate change compared with the level and the trend of the baseline data. The largest number of errors that occurred was multiple selections, averaging $0.88(S D=0.83)$. Erin's discriminative requesting during the generalization probes averaged $5.50(S D=4.04)$. The data points indicated a positive trend with high variability, and the levels were clustered around the upper side of the vertical axis. There were a few overlapping data points between the generalization and the baseline phases. During maintenance, Erin av- eraged $9.8(S D=0.50)$. The data points were high, and there was a slight decrease in trend with low variability and no overlap. The Tau- $U$ was 1.00 with $90 \% \mathrm{Cl}(0.40$, $1.00)$, which indicates a strong effect and statistical significance $(p=0.01)$.

2 symbols: During the baseline sessions, Erin's discriminative requesting averaged $0.75(S D=0.96)$. The data points were mainly in the lower level around the vertical axis and exhibited a slight positive trend and low variability. In the majority of the trials, Erin made incorrect responses by selecting the play symbol multiple times, averaging $2.5(S D=3.00)$. Erin met the acquisition criteria after the sixth session, and his performance averaged $8.17(S D=0.75)$. The data points exhibited a clear change in level with an upward trend, slight variability, and no overlap. There was an immediate change in the data points when delayed prompting was implemented after a 0 -s delay. During generalization probes, the data points averaged $9.17(S D=2.04)$, and they were high in level and exhibited a positive trend with low variability and no overlap. Erin was successful at using the iPad to request in all trials across all maintenance probes. The data points showed a high level and no trend, variability, or overlap. The Tau- $U$ effect size indicated a medium effect, 0.79 with $90 \% \mathrm{Cl}(0.15,1.00)$ and statistical significance $(p=0.00)$.

4 symbols: Erin used the iPad to request in a few trials during the baseline, and his averaged performance was $1.00(S D=1.15)$. The data points were low in their level around the $y$-axis and exhibited a slight upward trend with low variability. Erin frequently tapped on the play symbol repeatedly when his play was interrupted during the baseline phase, and his errors averaged 3.75 $(S D=4.35)$. For this field size, Erin met the acquisition criteria after the sixth session, and his performance averaged $8.33(S D=2.42)$. During the delayed-prompting condition, his data points were high and exhibited a positive trend with high variability and no overlap. There was an immediate change in the data points when the intervention was introduced. During generalization, Erin averaged $9.83(S D=0.41)$, and the data exhibited a high level with no trend, low variability, and no overlap. For the maintenance probes, independent discriminative request occurred in each trial across all probes. The data points were high in level and exhibited no trend, variability, or overlap. The Tau-U was 0.83 with $90 \% \mathrm{Cl}$ $(0.19,1.00)$, which indicates a medium effect and statistical significance $(p=0.03)$.

\section{Jacob}

1 symbol: The discriminative requesting did not occur during baseline. Jacob met the acquisition criteria after the $10^{\text {th }}$ session, and his performance averaged $7.00(S D=2.45)$. The data exhibited a gradual change in level, a positive trend with variable data points, and no overlap. There was an immediate change in the data 


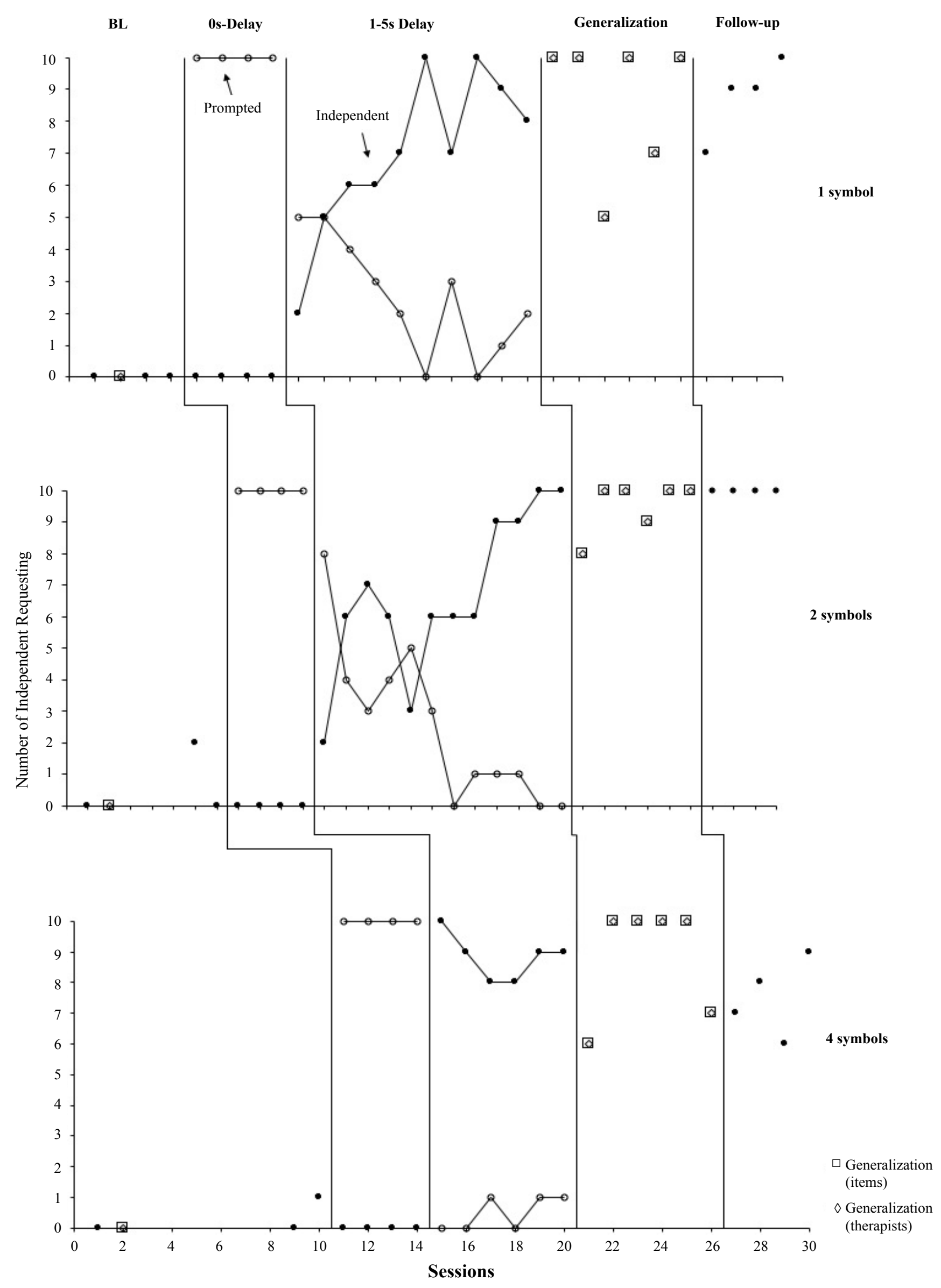

Figure 3: Number of independent requesting across different field sizes for Jacob.

points after implementing the prompt delay. Jacob was successful at using the iPad to request new toys across another behavioral therapist, and his performance averaged $7.83(S D=2.48)$. The data exhibited a high level, 
an upward trend with high variability, and no overlap. Jacob continued to use the iPad to request during the maintenance condition, and his performance averaged $8.75(S D=1.26)$. The data points were high in level and exhibited a positive trend with low variability and no overlap. The Tau- $U$ effect size was 1.00 with $90 \% \mathrm{Cl}$ $(0.42,1.00)$, which indicates a strong effect and statistical significance $(p=0.00)$.

2 symbols: Jacob selected the play symbol to request in a few trials in one baseline session. Jacob's performance averaged $0.50(S D=1.00)$. Frequently, Jacob initiated an incorrect discriminative request by tapping on the play symbol multiple times when his play was interrupted; his errors averaged $3.50(S D=4.12)$. During the delayed prompting, Jacob met the acquisition criteria after the $12^{\text {th }}$ session, and his performance averaged $6.67(S D=2.53)$. The data exhibited a gradual change in level, an upward trend with variable data points, and a few overlapping data points. There was pronounced change in the data points after the introduction of the delayed prompting. During the generalization probe, Jacob's performance averaged $9.50(S D=0.84)$, and the data points were high in level and exhibited a positive trend with slight variability and no overlap. Similarly, the discriminative request occurred in each trial across all the maintenance sessions. Therefore, the data points exhibited a high level, no trend, variability, and overlap. The Tau- $U$ effect size indicated a strong effect, 0.98 with $90 \% \mathrm{Cl}(0.41,1.00)$, and statistical significance $(p=0.00)$.

4 symbols: Independent use of the iPad to request occurred once in a baseline session and Jacob's performance averaged $0.25(S D=0.50)$. Jacob's most common error made was selecting the play symbol multiple items; his errors averaged $3.75(S D=4.79)$. During the delayed prompting condition, Jacob met the acquisition criteria after the third session, and his performance averaged $8.83(S D=0.75)$. The intervention data points exhibited a clear high level, no trend with low variability, and no overlap. There was an immediate change in the data points after the introduction of the delayed prompting. During the generalization probes, Jacob performance averaged $8.83(S D=1.83)$, and the data exhibited a high level, a positive trend, low variability, and no overlap. Jacob continued to use the iPad to request to play, and his performance averaged $7.50(S D=$ 1.29). The data points exhibited a high level, a positive trend with variability, and no overlap. The Tau-U was 0.88 with $90 \% \mathrm{Cl}(0.23,1.00)$, which indicates a medium effect and statistical significance $(p=0.03)$.

\section{Discussion}

The purpose of this study was to investigate the efficacy of applying NET to develop discriminative requesting in young children with ASD. The study also assessed whether the participants could generalize the acquired skills across novel stimuli. Per the visual analysis and the effect size measurement, the results indicated that NET was effective at teaching discriminative requesting of young children with ASD, which further supports the results of previous studies [18-20]. Indeed, several studies have demonstrated the effectiveness of incorporating naturalistic teaching approaches with low-tech SGDs in developing functional communication, operational, or strategical skills $[40,41]$.

Furthermore, the participants were successful at using the iPad to request across other stimuli (i.e., preferred items, behavioral therapists) that were not included in the training sessions. These results are consistent with those of previous studies in which the generalization of communication skills using SGDs was demonstrated across people and locations [41] as well as different preferred items [20]. Integrating various reinforcers (i.e., preferred items) and common communication partners that interact with the participants frequently throughout the day may have facilitated the generalization of the mands. Programming for generalization by incorporating common stimuli found in the natural environment is a well-established technique for developing the occurrence of the target behaviors in novel circumstances [42].

Regarding the modified procedure of PECS Phase III, the participants attained mastery across all fields of a different number of symbols, suggesting that the symbol discrimination training procedure was effective at teaching discriminative requesting. The participants required a different number of sessions to reach the acquisition criteria. For the fields of 1 and 2 symbols, they required an average of nine sessions to meet the acquisition criteria and an average of six sessions for the field of 4 symbols. Surprisingly, the number of distractor symbols in the display screen did not affect the participants' acquisition speed. In fact, the participants required fewer trials to meet the acquisition criteria when there were more distractor symbols compared with the other phases with fewer distractors. Our results support the previous research in which symbol discrimination training led to the acquisition of more complex discriminated mands $[19,27]$.

The primary factor that may have contributed to the rapid acquisition of discriminative requesting was giving the participants the opportunity to initiate a response by emitting behavior indicators. Behavior indicators-in the forms of reaching, pointing, gazing, or guiding someone's hand toward an item- occur in the presence of preferred items. These indicators communicate that the child is motivated to access these items [43]. The occurrence of behavior indicators helps to select the optimal opportunity to teach the child the appropriate form of communication. Child-initiated responses using high-tech AAC modalities have shown to develop functional communication skills $[40,41]$. In these studies, the participants were given an opportunity to request only when they showed an interest in an item, which 
ensured that their response was under the control of motivating operation to be a true request [44].

The positive outcomes of the study are most likely due to the use of a single symbol that, upon activation, produced a general request to play with several preferred items. The decision to not include a specific icon for each preferred item stemmed from the participants' preferences changing over time, which made it difficult to vary the number of included symbols throughout the study. One of the primary advantages of teaching generic or general is that this method permits the participants to encounter different preferred items when selecting a single symbol [45]. In addition, enriching the environment with participants' current preferred toys helps to facilitate stimulus generalization, increase ecological validity, and maintain motivation or interest to request preferred items.

The participants showed rapid acquisition of discriminative requesting using the iPad compared to the difficulties they faced when using PECS. Other factors could have contributed to their difficulties, such as inconsistency or inaccuracy in implementation. Response effort may be the reason behind the rapid acquisition of augmented requesting. Johnston, Reichle, and Evans (2004) [46] have discussed factors that determine the efficiency of alternative communication form, response effort, a schedule of reinforcement, an immediacy of reinforcement, and quality of reinforcement. Response effort refers to the physical, emotional, and cognitive demands that a response requires from the individual. The alternative verbal behavior must be equal or less in response effort for the individual to engage in this response. For some children with ASD, activating a symbol on a device is less effortful compared to exchanging a picture to access a preferred item. In a study conducted by [47], the investigators compared between two different requesting topographies (iPad and picture exchange-PE) in terms or acquisition and preference. The results indicated that the participants showed preference and increased rates of requesting using the iPad. The investigators mentioned that response effort might be one of the factors that contributed to the surpassing of the iPad compared to PE.

Moreover, iconicity-the correspondence between the symbol and its referent-plays a vital role in symbol learnability [48]. Fuller (1997) [49] described the degree of iconicity in three distinctly different levels as transparent (i.e., highly iconic), translucent (i.e., moderately iconic), and opaque (i.e., little to no iconicity). Considerable evidence suggests that symbols that strongly resemble their referents are more learned easily compared with symbols with a lower iconicity level [50]. Despite the iconicity hypothesis, the participants in the current study learned to select a translucent symbol (i.e., the play icon) to grant access to various preferred toys. The results are consistent with those of a previous study in which the participants exhibited no difference in their ability to use transparent or translucent symbols to request preferred items [51].

Overall, the results of this study, albeit preliminary, support and extend the existing literature evaluating the effects of naturalistic teaching approaches in the development of discriminative requesting using high-tech SGDs. Future research needs to replicate the findings across individuals with ASD with varying characteristics.

\section{Limitations and Future Research}

Several limitations should be considered when interpreting the results of this study. First, even though the participants were similar in their clinical and demographical characteristics, the small sample size prevents the generalization of the results to other young children with ASD. Second, because the investigation was limited to general single-step requesting, future studies should investigate whether symbol discrimination training would facilitate the development of advanced discriminative social-communication skills. In addition, since the field size was limited to 4 symbols, future research should also explore several factors pertaining to the display design, such as the number, location, size, and the level of iconicity of the symbols [48] to further determine the effectiveness of symbols' discrimination training. Further, since the intervention was implemented using multiple-probe design across field sizes, there was a possibility that the results were affected by carry-over effect due to the fact the different number of symbols in each field size was not independent. Therefore, the participants' performance in the field with 2 symbols might have been affected by the previous exposure of a single symbol field size. Future studies are recommended to replicate the results using more rigorous experimental design (e.g., changing-criterion design).

\section{Funding}

This research did not receive any specific grant from funding agencies in the public, commercial, or not-forprofit sectors.

\section{Conflict of Interest}

The authors declare that they have no conflict of interest.

\section{References}

1. American Psychiatric Association (2013) Diagnostic and statistical manual of mental disorders (DSM-5). American Psychiatric Publishing, Arlington, VA, USA.

2. Ganz JB, Earles-Vollrath TL, Heath AK, Parker RI, Rispoli MJ, et al. (2012) A meta-analysis of single case research studies on aided augmentative and alternative communication systems with individuals with autism spectrum disorders. J Autism Dev Disord 42: 60-74.

3. Light JC, Roberts B, Dimarco R, Greiner N (1998) Augmentative and alternative communication to support receptive and expressive communication for people with autism. Journal of Communication Disorders 31: 153-180. 
4. Schlosser RW, Sigafoos J, Koul RK (2009) Speech output and speech-generating devices in autism spectrum disorders. In: P Mirenda, T lacono, J Light, AAC for Individuals with Autism Spectrum MD: Paul H, Brookes Disorders. Baltimore, USA, 141-169.

5. Rispoli MJ, Franco JH, van der Meer L, Lang R, Camargo SPH (2010) The use of speech-generating devices in communication interventions for individuals with developmental disabilities: A review of the literature. Dev Neurorehabil 13: 276-293.

6. Schlosser RW, Koul RK (2015) Speech output technologies in interventions for individuals with autism spectrum disorders: A scoping review. Augment Altern Commun 31: 285-309.

7. Lorah ER, Parnell A, Whitby PS, Hantula D (2014) A systematic review of tablet computers and portable media players as speech generating devices for individuals with autism spectrum disorder. J Autism Dev Disord 45: 37923804 .

8. Kagohara DM, van der Meer L, Achmadi D, Green VA, O'Reilly MF, et al. (2012) Teaching picture naming to two adolescents with autism spectrum disorders using systematic instruction and speech-generating devices. Research in Autism Spectrum Disorders 6: 1224-1233.

9. Lorah ER, Parnell A, Speight DR (2014) Acquisition of sentence frame discrimination using the $\mathrm{iPad} \circledast$ as a speech-generating device in young children with developmental disabilities. Research in Autism Spectrum Disorders 8: $1734-1740$

10. Lorah ER, Karnes A, Speight DR (2015) The acquisition of intraverbal responding using a speech-generating device in school aged children with autism. Journal of Developmental and Physical Disabilities 27: 557-568.

11. Mirenda P (2003) Toward functional augmentative and alternative communication for students with autism manual signs, graphic symbols, and voice output communication aids. Language, Speech, and Hearing Services in Schools 34: $203-216$

12. Logan K, lacono T, Trembath D (2017) A systematic review of research into aided AAC to increase social-communication functions in children with autism spectrum disorder. Augment Altern Commun 33: 51-64.

13. Sundberg ML, Partington JW (1999) The need for both discrete trial and natural environmental language training for children with autism. In: PM Ghezzi, WL Williams, JE Carr, Autism: Behavior analytic perspectives. Context Press, Reno, Nevada, USA, 139-156.

14. Koegel RL, O'dell MC, Koegel LK (1987) A natural language teaching paradigm for nonverbal autistic children. $J$ Autism Dev Disord 17: 187-200.

15. Koegel RL, Koegel LK, Surratt A (1992) Language intervention and disruptive behavior in preschool children with autism. J Autism Dev Disord 22: 141-153.

16. Sundberg ML, Loeb M, Hale L, Eigenheer P (2002) Contriving establishing operations to teach mands for information. Anal Verbal Behav 18: 15-29.

17. Hall G, Sundberg ML (1987) Teaching mands by manipulating conditioned establishing operations. Anal Verbal Behav 5: 41-53.

18. Flores M, Musgrove K, Renner S, Hinton V, Strozier S, et al. (2012) A comparison of communication using the Apple $\mathrm{iPad}$ and a picture-based system. Augment Altern Commun 28: $74-84$.
19. Lorah ER (2018) Evaluating the iPad mini as a speech-generating device in the acquisition of a discriminative mand repertoire for young children with autism. Focus on Autism and Other Developmental Disabilities 33: 47-54.

20. Sigafoos J, Lancioni GE, O'Reilly MF, Achmadi D, Stevens $M$, et al. (2013) Teaching two boys with autism spectrum disorders to request the continuation of toy play using an iPad-based speech-generating device. Research in Autism Spectrum Disorders 7: 923-930.

21. Ganz JB (2015) AAC Interventions for individuals with autism spectrum disorders: State of the science and future research directions. Augment Altern Commun 31: 203-214.

22. Still K, Rehfeldt RA, Whelan R, May R, Dymond S (2014) Facilitating requesting skills using high-tech augmentative and alternative communication devices with individuals with autism spectrum disorders: A systematic review. Research in Autism Spectrum Disorders 8: 1184-1199.

23. Achmadi D, Sigafoos J, van der Meer L, Sutherland D, Lancioni GE, et al. (2014) Acquisition, preference, and follow-up data on the use of three AAC options by four boys with developmental disability/delay. Journal of Developmental and Physical Disabilities 26: 565-583.

24. Green G (2001) Behavior analytic instruction for learners with autism: Advances in stimulus control technology. Focus on Autism \& Other Developmental Disabilities 16: 7285.

25. Williams G, Donley CR, Keller JW (2000) Teaching children with autism to ask questions about hidden objects. J Appl Behav Anal 33: 627-630.

26. Clark KM, Green G (2004) Comparison of two procedures for teaching dictated-word/symbol relations to learners with autism. J Appl Behav Anal 37: 503-507.

27. Lorah ER, Crouser J, Gilroy SP, Tincani M, Hantula D (2014) Within stimulus prompting to teach symbol discrimination using an iPad speech-generating device. Journal of Developmental and Physical Disabilities 26: 335-346.

28. Sundberg M (2008) The Verbal Behavior: Milestones assessment and placement program. AVB Press, Concord, California, USA.

29. Gilliam JE (2013) Gilliam autism rating scale: GARS. (3 ${ }^{\text {rd }}$ edn), Pro-ed, Inc, Austin, Texas, USA.

30. Harrison PL, Oakland T (2003) Adaptive behavior assessment system. ( $2^{\text {nd }}$ edn), The Psychological Corporation, San Antonio, USA.

31. Gibson EJ (1973) Principles of perceptual learning and development. Appleton-Century-Crofts, New York, USA.

32. Schreibman L, Dawson G, Stahmer AC, Landa R, Rogers SJ, et al. (2015) Naturalistic developmental behavioral interventions: Empirically validated treatments for autism spectrum disorder. J Autism Develop Disord 45: 2411-2428.

33. Barlow DH, Andrasik F, Hersen M (2006) Single-case experimental designs. ( $3^{\text {rd }}$ edn), Allyn \& Bacon, New York, USA.

34. Horner RD, Baer DM (1978) Multiple-probe technique: A variation on the multiple baseline. J Appl Behav Anal 11: 189-196.

35. Assistive Ware (2016) Proloquo2Go ${ }^{\mathrm{TM}}$.

36. Green VA, Sigafoos J, Didden R, O'Reilly MF, Lancioni GE, et al. (2008) Validity of a structured interview protocol for assessing children's preferences. In: P Grotewell, Y Burton, Early childhood education: Issues and developments. Nova Science Publishers, New York, USA, 87-103. 
37. Roane HS, Vollmer TR, Ringdahl JE, Marcus BA (1998) Evaluation of a brief stimulus preference assessment. $J$ Appl Behav Anal 31: 605-620.

38. Duker P, Didden R, Sigafoos J (2004) One-to-one training: Instructional procedures for learners with developmental disabilities. Pro-Ed Inc, Austin, Texas, USA.

39. Parker R, Vannest KJ, Davis JL, Sauber S (2011) Combining non-overlap and trend for single case research: Tau-U. Behav Ther 42: 284-299.

40. Schepis MM, Reid DH, Behrmann MM, Sutton KA (1998) Increasing communicative interactions of young children with autism using a voice output communication aid and naturalistic teaching. Journal of Applied Behavior Analysis 31: $561-578$.

41. Sigafoos J, O'Reilly M, Seely-York S, Edrisinha C (2004) Teaching students with developmental disabilities to locate their AAC device. Res Dev Disabil 25: 371-383.

42. Stokes T, Baer D (1977) An implicit technology of generalization. J Appl Behav Anal 10: 349-367.

43. Drasgow E, Halle JW, Sigafoos J (1999) Teaching communication to learners with severe disabilities: Motivation, response competition, and generalization. Australasian Journal of Special Education 23: 47-63.

44. Skinner BF (1957) Verbal behavior. Appleton-Century-Crofts, East Norwalk, Connecticut, USA.

45. Sigafoos J, Reichle J (1992) Comparing explicit to generalized requesting in an augmentative communication mode.
Journal of Developmental and Physical Disabilities 4: 167188.

46. Johnston S, Reichle J, Evans J (2004) Supporting augmentative and alternative communication use by beginning communicators with severe disabilities. Am J Speech Lang Pathol 13: 20-30.

47. Torelli JN, Lambert JM, Da Fonte MA, Denham KN, Jedrzynski TM, et al. (2016) Assessing acquisition of and preference for mand topographies during functional communication training. Behav Anal Pract 9: 165-168.

48. Lloyd LL, Fuller D (1990) The role of iconicity in augmentative and alternative communication symbol learning. In: W Fraser, Key issues in mental retardation research. Routledge, London, UK, 295-306.

49. Fuller DR (1997) Initial study into the effects of translucency and complexity on the learning of Blissymbols by children and adults with normal cognitive abilities. AAC: Augmentative and Alternative Communication 13: 30-39.

50. Koul RK, Schlosser RW, Sancibrian S (2001) Effects of symbol, referent, and instructional variables on the acquisition of aided and unaided symbols by individuals with autism spectrum disorders. Focus on Autism and Other Developmental Disabilities 16: 162-176.

51. Angermeier K, Schlosser RW, Luiselli JK, Harrington C, Carter B (2008) Effects of iconicity on requesting with the picture exchange communication system in children with autism spectrum disorder. Research in Autism Spectrum Disorders 2: 430-446. 